\title{
Kinematic compatibility of a wrist robot with cable differential actuation: effects of misalignment compensation via passive joints
}

\author{
Haider A. Chishty, Andrea Zonnino, Andria J. Farrens, Fabrizio Sergi, IEEE Member
}

\begin{abstract}
We present the UDiffWrist (UDW), a low-impedance 2-DOF wrist exoskeleton featuring a cable-differential transmission. To investigate the effect of different design strategies for achieving kinematic compatibility, we developed two versions of this robot: One version (UDW-C) achieves kinematic compatibility only in the case of perfect alignment between human and robot joints. The second version (UDW-NC) connects the human and robot via passive joints to achieve kinematic compatibility regardless of alignment between human and robot joints. Through characterization experiments, we found that the UDW-NC was more robust to misalignments than the UDW-C: the increase in maximum interaction torque associated with misalignments was greater for the UDW-C than the UDW-NC robot $(p=0.003)$. However, the UDW-NC displayed greater Coulomb friction $(p<0.001)$. Further, Coulomb friction increased more for the UDW-NC than the UDW-C in the presence of misalignments between the human and robot axes $(p<0.001)$. We also found that torque transfer was more accurate in the UDW-C than in the UDW-NC. These results suggest that for the small (10 deg) 2-DOF wrist movements considered, the advantages of the UDW-NC in terms of kinematic compatibility are likely overshadowed by the negative effects in friction and torque transfer accuracy.
\end{abstract}

Index Terms-Kinematic compatibility, exoskeleton, misalignments, wrist, impedance.

\section{INTRODUCTION}

$\mathrm{W}$ EARABLE robots have a variety of applications in rehabilitative and assistive settings, and are increasingly used by individuals with neuromotor disorders [1], [2]. In rehabilitation, it has been shown that movement therapy based on repetitive and high-intensity motor practice has yielded positive effects on motor function and recovery [3]-[8]. However, the longer sessions necessary to achieve the necessary movement repetitions can fatigue therapists [9]. Robots are ideal tools to support movement therapy as they can produce consistent mechanical actions that can measure, assist, resist, or perturb patient movements, relieving the physical burden on the therapist and allowing them to oversee the session. Indeed, there is evidence that robotic sensorimotor training improves motor performance relative to standard therapeutic methods [7], [10]-[12].

Wearable devices for rehabilitation of upper-limb movements can be categorized into two groups: end-effector type robots and exoskeletons. Exoskeletons, which run parallel to the arm and thus require more than one point of contact, offer unique benefits due to their ability to manipulate and measure

Corresponding authors: F.S. (fabs@udel.edu). The work was done when the authors were all with the Human Robotics Laboratory, Department of joint torques and angles directly [13], [14]. This capability is critical in rehabilitation settings, where having direct control over specific degrees of freedom (DOFs) of the limb allows for targeted interventions [11], [12]. To correctly target individual joints, however, it is crucial that exoskeletons do not impose restrictions on the user's motion, a condition referred to as kinematic compatibility [15].

Anthropomorphic exoskeletons typically attempt to replicate the kinematics of the targeted human limb [17]. However, in order for these robots to be kinematically compatible with users, they must perfectly replicate the human kinematics of the limb, such that the axes of rotation of the robotic joints perfectly align with those of the human joints [10], [14]. However, perfect alignment is impossible to achieve in practice: the complexity of joint geometry, uncertainty of precise joint location, and slippage of the robot-user connection complicate the alignment [9], [18]. The resulting misalignments induce parasitic interaction forces between the robot and user which can lead to pain, discomfort, and damage to the user's skin and joints [9], [17].

Kinematic compatibility can be improved by fully mimicking the complex kinematics of the targeted human joints. For example, to account for the non-stationary nature of the shoulder's axis of rotation, the Harmony exoskeleton [19] implements a novel shoulder mechanism, comprised of a revolute joint and four-bar mechanism in series, that follows the same trajectory as the shoulder. Similarly, the ARMin III [20] demonstrates a novel shoulder actuation principle based on minimizing the distance between the robot and shoulder's axis of rotation over the workspace, allowing for optimal placement of robotic joints.

The above strategies complicate the kinematic structure of these systems. A more general solution, implemented in previous iterations of the ARMin [13], [21] and expanded on in the ARMin III, is to introduce passive DOFs within the robotic chain to achieve kinematic redundancy. The result is a system that self-adjusts, ensuring that axes remain aligned between user and robot. Common implementations include the incorporation of passive linear joints to decouple joint rotations and translations [17], [18]. To account for possible angular misalignments between robot and user, a passive revolute joint can be additionally included to form a revolute-revoluteprismatic (RRP) joint configuration. This strategy has been used in both upper and lower limb exoskeletons [22], [23].

Biomedical and Mechanical Engineering, University of Delaware, Newark, DE 19713 USA. A.Z. is now with Haexel Robotics, Rome, Italy. 
An alternate method introduced in [9] and expanded on in [17], [24], and [25] forgoes the need of the robot to imitate human kinematics, bypassing the alignment requirement, and instead achieves kinematic compatibility by providing a bridging mechanism between exoskeleton and user. This strategy connects the two serial chains (the human limb and the exoskeleton) via a set of fixations comprised of passive joints. These passive fixations serve to minimize hyperstatic interaction forces between robot and user. This method is capable of significantly reducing interaction loads [17]. The benefit of this strategy over the self-aligning method is that the kinematic chain of the robot is untouched, thus not overcomplicating its structure and control.

While the above two categories solve the issue of misalignment, there are drawbacks associated with the addition of passive joints. First, without extra active components, these mechanisms are fully passive; as such, they may displace under load and prevent the transfer of large torques through their structure and to the desired human joints [15], [26], [27]. Second, the friction and inertia introduced by the passive mechanisms are intrinsic shortcomings, as these heavily affect the transparency of the system. In fact, as discussed in [28], increased inertia has a significantly greater effect on gait kinematics than misalignment.

The need for accurate torque transfer and low impedance is particularly relevant for the rehabilitation of the wrist [29]. In addition to the aforementioned importance of specific joint control, the effective friction, inertia, and stiffness of the system should be low enough that the user can easily backdrive the system [29]. As such, typical wrist robots have focused on low inertia, low friction designs: The RiceWrist [30] and MRSoftWrist [31] implement parallel designs via 3-RPS (revoluteprismatic-spherical) platforms, resulting in not only lower inertia, but also higher torque output. In contrast, the robot used in [32] implements a serial structure with direct drive motors. The RiceWrist-S [33] and OpenWrist [34] also use serial structures, but incorporate RRR (revolute-revolute-revolute) joint configurations with remote cable actuation to ensure low friction and backlash. The transmissions are placed proximal on the limb to reduce inertia. The WRES [35] uses a similar cable actuation via a tendon-driven differential transmission resulting in a compact and low inertia system, with low friction and negligible backlash [36]. This transmission allows for a backdrivable system, and has been used in the design of humanoid robotics [37], surgery [38], rehabilitation [39], and gravity counterbalancing [40].

While some of the above wrist systems also feature passive linear joints to address possible misalignments, these mechanisms do not compensate for both position and orientation misalignment between the users' and robot wrist in all DOFs. In fact, using the methodology described in [17], we can conclude that all such systems are hyperstatic, and thus theoretically not kinematically compatible. To the authors' knowledge, the implementation of kinematic design strategies for wrist exoskeletons to achieve full kinematic compatibility using alignment-free approaches has not yet been attempted. In fact, the inclusion of enough passive joints necessary to achieve isostaticity is usually omitted for the sake of reduced device complexity [41]. The reduced backdrivability associated with the frictional and inertial effects of the added passive mechanism further lends to the argument for their omission.

As identified above, there is a trade-off between two conflicting objectives of exoskeleton design: 1) ensuring low endpoint impedances; and 2) minimizing the effect of misalignments [28]. This trade-off has not been extensively studied for wrist robots, and it is thus unclear how necessary the inclusion of a misalignment compensation mechanism is. To validate the efficacy of an alignment-free approach in a wrist exoskeleton, a high transparency system must be developed and validated.

We present here the development and characterization of the UDiffWrist (UDW), a novel 2-DOF wrist exoskeleton that features a cable differential transmission. The cable differential allows for a low inertia/friction system that results in low output impedance. We present two versions of this device that differ based on the approach used to achieve kinematic compatibility. One version, (UDW-C), achieves kinematic compatibility only in the presence of perfect alignment between robot and anatomical joints. The second version, (UDW-NC), connects the human and robot kinematic chains via a set of passive joints to achieve kinematic compatibility regardless of alignment between robot and anatomical joints. We quantify the effects of different robot designs on backdrivability, torque transfer capability, and robustness to misalignments via dynamic characterization experiments conducted on both devices.

\section{DESIGN AND MODELLING}

The UDiffWrist is a two DOF wrist robot that tracks and supports movements in flexion extension (FE) and radial/ulnar deviation (RUD) by means of a cable differential transmission. The use of a differential allows the transmission to be placed at the proximal end of the robot's kinematic chain to reduce the overall inertia of the system. Cable actuation allows the system to be low friction and backlash-free.

The cable differential transmission provides rotations about two orthogonal axes, intersecting at a single point. This differs from the kinematics of the wrist joint, as the axes of FE and RUD rotation are not exactly orthogonal [42], [43], and have a non-zero offset. To investigate any kinematic incompatibilities that may arise due to this fact, two different designs have been developed: a design that assumes perfect joint alignment between user and system after attempting to co-locate both sets of joints; and an alignment-free design that implements a passive mechanism between the robotic and human chain, and therefore does not require any co-location. In this paper, these designs will be referred to as the UDiffWrist-Colocated (UDWC) and UDiffWrist-NonColocated (UDW-NC) respectively.

The two joints of the UDW-C are intended to directly correspond to the RUD and FE DOFs of the human wrist, which is placed inside the transmission. The system features three Aluminum 6061 pulleys routed together using stainless steel coated wires to form the cable differential transmission. The differential is held by a three-part carrier also made of Aluminum 6061. The output shaft and end-effector of the system are fabricated using an SLA 3D printer (Form 3, Formlabs). The entire system is mounted in an Aluminum 6061 housing (Fig. 1A,B,E). 


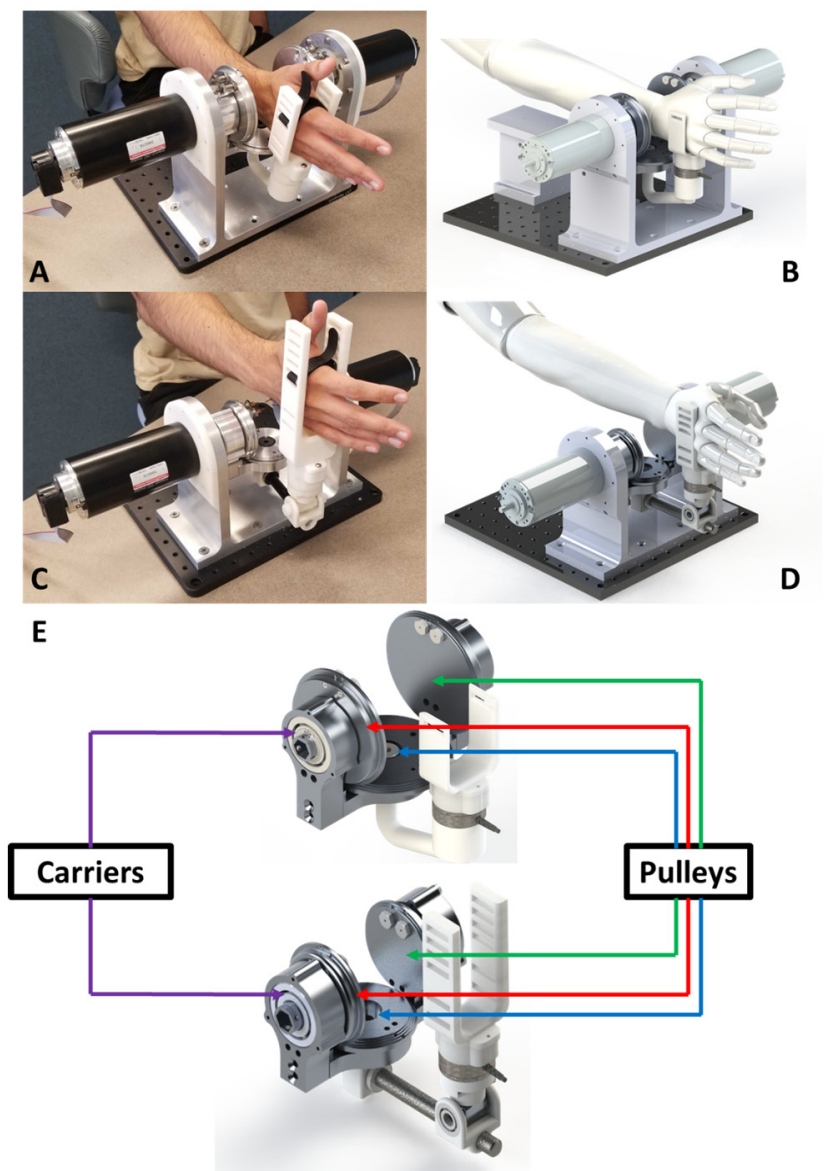

Fig. 1. Pictures and 3D CAD renders of the two versions of the UDW. Users locate their wrist within the transmission of the UDW-C (A and B), and above the transmission of the UDW-NC (C and D). (E) shows the pulleys and carriers of the two transmissions (top: UDW-C, bottom: UDW-NC).

The UDW-NC includes several passive joints to achieve kinematic compatibility. The robotic and human chains are bridged using a 4 DOF passive mechanism comprised of three revolute and one prismatic joint. Given the presence of passive joints, there is no need to align the axes of rotation of the transmission with the anatomical axes of the wrist of the user. As such, users grasp the handle of the UDW-NC with their wrist above the transmission. Relaxing the alignment constraint, in fact, allows for the reduction of the transmission dimensions: the transmission, carrier, and housing are smaller than those of the UDW-C, though they are made of the same materials. Further, the passive mechanism of the system is made of SLA printed components, bearings, and a carbon steel rod (Fig. 1C,D,E).

Both systems are actuated by two RE 65 brushed motors (Maxon Precision Motors, Sachseln, Switzerland, nominal torque: $501 \mathrm{mNm}$, stall torque: $15700 \mathrm{mNm}$ ), including AEDL5810-Z13 optical encoders with 5000 counts/turn quadrature resolution. Interaction forces are quantified via a 6 -axis force transducer ATI Mini40 F/T Sensor (ATI Industrial Automation Inc., Apex, NC, USA) located at the end-effector. Motors were driven by Escon 70/10 Servo Controllers (Maxon Precision Motors, Sachseln, Switzerland). Data was recorded using a Q8USB data acquisition card (Quanser Consulting, Markham, ON, Canada). Control was implemented in MATLAB 2017a (The

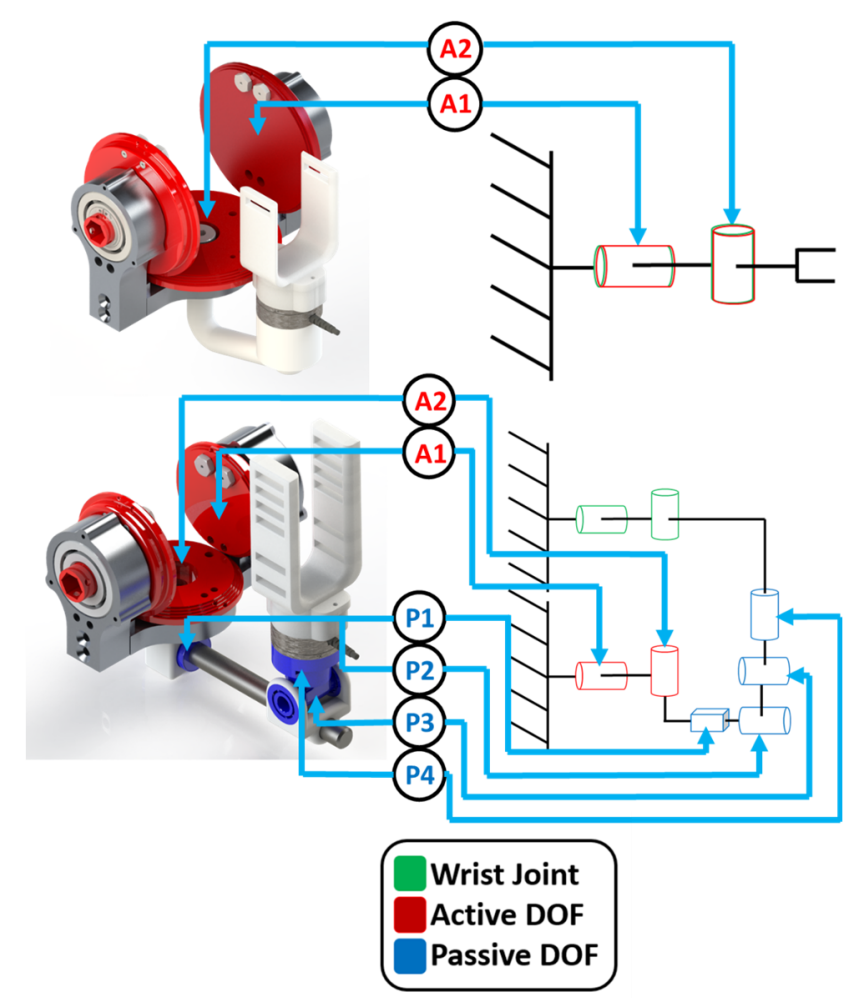

Fig. 2. CAD models and kinematic schemes of robot transmissions: UDW-C (top); UDW-NC (bottom). Matching joints are labeled: Active DOFs are the outputs of the cable differential transmission. In the UDW-C design, the two sets of active and wrist DOFs are assumed to be perfectly co-located, and thus overlap.
A

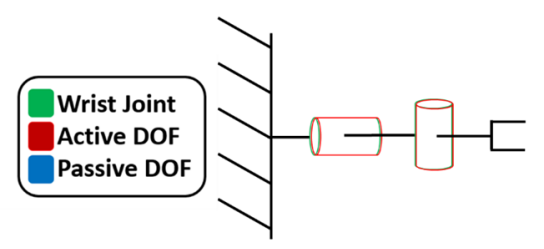

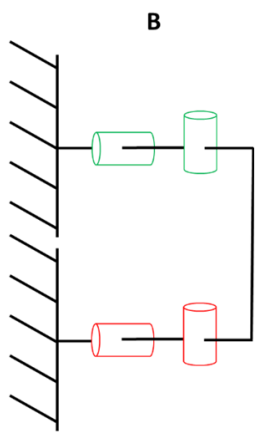

Fig. 3. UDW-C kinematic scheme with assumed perfect alignment (A) and without (B). Without the assumed alignment, the transmission and wrist DOFs no longer collapse, resulting in different mechanism mobility.

MathWorks Inc., Natick, MA, USA) using the Quarc real-time engine (Quanser Consulting, Markham, ON, Canada).

\section{A. Mobility Analysis \\ 1) $U D W-C$}

Under perfect alignment, the two degrees of freedom of the human wrist overlap with the two active degrees of freedom of the robot, resulting in a serial chain with 2 DOFs (Fig. 2, Fig. $3 \mathrm{~A})$. However, if the human and robot joints are not aligned (Fig. 3B), the mobility of the system can be found using Grubler's Mobility Criterion seen in (1):

$$
M=6(N-1-j)+\sum_{i=1}^{j} f_{i}
$$




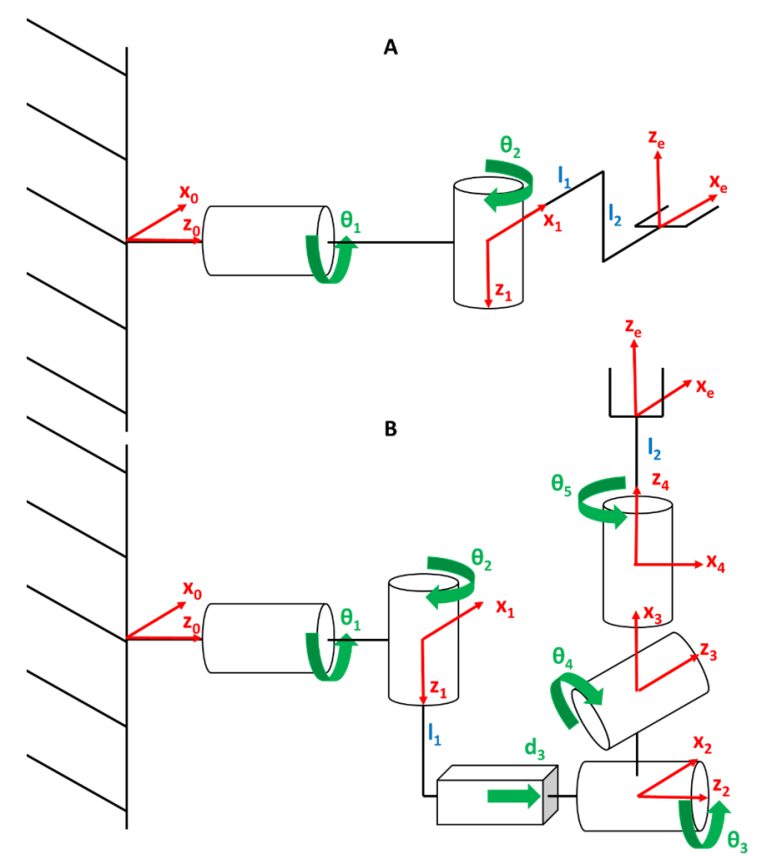

Fig. 4. Zero configurations of UDW-C (A) and UDW-NC (B). Joint frames and variables are labelled. Non-labelled links are zero length. These zero configurations were used to derive the D-H parameters of the systems.

Where $N$ is the number of links in the system comprising both human and robot segments, $j$ is the number of joints, and $f_{i}$ is the degrees of freedom of joint $i$. Using this criterion $(N=4, j$ $\left.=4, f_{i}=1\right)$, the studied system has -2 degrees of freedom, i.e., the system is hyperstatic. As such, in the case of misalignments, the UDW-C should not allow for the two desired independent rotations of the FE and RUD joints.

\section{2) $U D W-N C$}

To resolve the kinematic limitations of the UDW-C, the UDW-NC includes four passive degrees of freedom added via a mechanism connecting the robot and human kinematic chains (Fig. 2). The resulting system $\left(N=8, j=8, f_{i}=1\right)$ has 2 degrees of freedom, as desired, regardless of the alignment between the axes of human and robot joints.

\section{B. Kinematics}

The differential transmission serves as the first two joints in the kinematic chain of both designs. For a cable differential system, the transformations between the rotations of the two motors $\left(e_{1}\right.$ and $\left.e_{2}\right)$ and the rotations of the two output joints $\left(j_{1}\right.$ and $j_{2}$ ) are given by (2) and (3).

$$
\begin{gathered}
\theta_{j_{1}}=\frac{\theta_{e_{1}}-\theta_{e_{2}}}{2} \\
\theta_{j_{2}}=\frac{\theta_{e_{1}}+\theta_{e_{2}}}{2} \\
\text { TABLE } 1
\end{gathered}
$$

D-H PARAMETERS - UDW-C

\begin{tabular}{ccccc}
\hline \hline$i$ & $d_{i}$ & $\theta_{i}$ & $a_{i}$ & $\alpha_{i}$ \\
\hline 1 & 0 & $\theta_{1}$ & 0 & $\frac{\pi}{2}$ \\
2 & $l_{2}$ & $\theta_{2}$ & $l_{1}$ & $\pi$ \\
\hline \hline
\end{tabular}

\section{1) $\mathrm{UDW}-\mathrm{C}$}

Due to the assumed co-location of the system and wrist axes of rotation, the two joints of the UDW-C robotic chain directly correspond to the joints of the wrist. As such $\theta_{R U D}=\theta_{l}$ and $\theta_{F E}$ $=\theta_{2}$ (Table 1$)$.

\section{2) $U D W-N C$}

The forward kinematics of the UDW-NC required more computation than the UDW-C. The first step was to derive the transformations between all relevant frames using the DenavitHartenberg parameters (Table 2), found from the zeroconfiguration of the robot (Fig. 4B). Then, transformation matrices between frames were found using (4).

$$
A_{i}^{i-1}=\left[\begin{array}{cccc}
c_{\theta_{i}} & -s_{\theta_{i}} c_{\alpha_{i}} & s_{\theta_{i}} s_{\alpha_{i}} & a_{i} c_{\theta_{i}} \\
s_{\theta_{i}} & c_{\theta_{i}} c_{\alpha_{i}} & -c_{\theta_{i}} s_{\alpha_{i}} & a_{i} s_{\theta_{i}} \\
0 & s_{\alpha_{i}} & c_{\alpha_{i}} & d_{i} \\
0 & 0 & 0 & 1
\end{array}\right]
$$

The overall transformation matrix of the system was determined using these matrices. As only the joint positions of Joints 1 and 2 are measured (the joints corresponding to the transmission; positions are found using (2) and (3), respectively), positions of joints 3 through 6 were estimated using non-linear least-squares optimization. During user interaction with the robot, the system has two distinct kinematic chains (human and robot) that eventually meet at the endeffector. Given a desired end-effector orientation (and thus a desired joint configuration $\theta_{R U D}$ and $\theta_{F E}$ for the human chain), the optimizer calculates the only joint configuration for the robotic chain that produces the same end-effector orientation.

However, as it is computationally intensive to perform this optimization online, third-order polynomial models (based on the known joint positions of joint 1 and joint 2) were fit to the

\begin{tabular}{|c|c|c|c|c|}
\hline \multicolumn{5}{|c|}{$\begin{aligned} \theta_{R U D}= & a_{1}+b_{1} \theta_{j_{1}}+c_{1} \theta_{j_{2}}+d_{1} \theta_{j_{1}}^{2}+e_{1} \theta_{j_{2}}^{2}+f_{1} \theta_{j_{1}} \theta_{j_{2}} \\
& +g_{1} \theta_{j_{1}}^{3}+h_{1} \theta_{j_{2}}^{3}+k_{1} \theta_{j_{1}}^{2} \theta_{j_{2}}+l_{1} \theta_{j_{1}} \theta_{j_{2}}^{2} \\
\theta_{F E}= & a_{2}+b_{2} \theta_{j_{1}}+c_{2} \theta_{j_{2}}+d_{2} \theta_{j_{1}}^{2}+e_{2} \theta_{j_{2}}^{2}+f_{2} \theta_{j_{1}} \theta_{j_{2}} \\
& +g_{2} \theta_{j_{1}}^{3}+h_{2} \theta_{j_{2}}^{3}+k_{2} \theta_{j_{1}}^{2} \theta_{j_{2}}+l_{2} \theta_{j_{1}} \theta_{j_{2}}^{2}\end{aligned}$} \\
\hline \multicolumn{5}{|c|}{$\begin{array}{c}\text { TABLE } 2 \\
\text { D-H PARAMETERS - UDW-NC } \\
\end{array}$} \\
\hline$i$ & $d_{i}$ & $\theta_{i}$ & $a_{i}$ & $\alpha_{i}$ \\
\hline 1 & 0 & $\theta_{1}$ & 0 & $\frac{\pi}{2}$ \\
\hline 2 & $l_{1}$ & $\theta_{2}$ & 0 & $-\frac{\pi}{2}$ \\
\hline 3 & $d_{3}$ & $\theta_{3}+\frac{\pi}{2}$ & 0 & $\frac{\pi}{2}$ \\
\hline 4 & 0 & $\theta_{4}+\frac{\pi}{2}$ & 0 & $\frac{\pi}{2}$ \\
\hline 5 & $l_{2}$ & $\theta_{5}+\frac{\pi}{2}$ & 0 & 0 \\
\hline
\end{tabular}
outputs of this analysis and used for simplified forward kinematic estimations. Models were created for estimating $\theta_{R U D}$ and $\theta_{F E}$ independently, as seen in (5) and (6). 

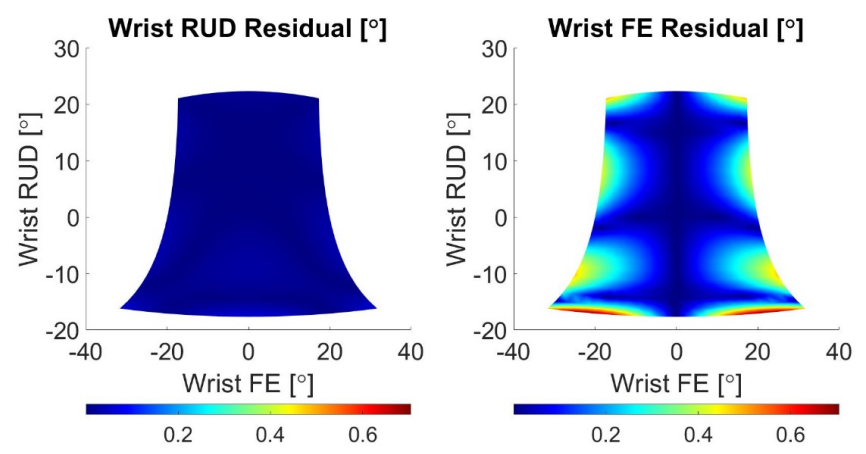

Fig. 5. Residual plots of forward kinematic fits of wrist RUD (left) and wrist $\mathrm{FE}$ (right). $\mathrm{X}$ axes denote true wrist position in FE; $\mathrm{Y}$ axes denote true wrist position in RUD.

In the admissible workspace, the average residual of the fit for $\theta_{R U D}$ was $0.011^{\circ}$ (maximum: $0.066^{\circ}$ ), and for $\theta_{F E}$ was $0.156^{\circ}$ (maximum: $0.705^{\circ}$ ) (Fig. 5). These low fit errors validate the use of polynomial fitting for the estimate of the wrist joint angles.

\section{Joint-Space Kinetics}

The kineto-static duality of the system was used to convert loads seen by the force transducer at the end-effector to wrist torques. The Jacobian of the system was found via (7), where $z_{0}$ is the axis of rotation of the wrist RUD joint, $z_{1}$ is the axis of rotation of the wrist FE joint, and $p_{e}$ is the position of the endeffector (hand) in the world frame. From here, joint torques were found using (8), where $\gamma_{\text {world }}$ are end-effector loads as seen from the world frame.

$$
\begin{gathered}
J=\left[\begin{array}{cc}
Z_{0} \times p_{e} & z_{1} \times p_{e} \\
z_{0} & z_{1}
\end{array}\right] \\
\tau=J^{T} \gamma_{\text {world }}
\end{gathered}
$$

\section{CHARACTERIZATION}

\section{A. Dynamic Transparency}

The dynamic transparency of both versions of the UDiffWrist was quantified during movements executed along eight trajectories within the system's workspace (pure FE, pure RUD, and their combinations, in positive and negative directions - Fig. 6). For each trajectory, transparency was quantified via three measures: Coulomb friction $\tau_{C}(\mathrm{~N} \cdot \mathrm{m})$, viscous friction $b(\mathrm{~N} \cdot \mathrm{m} \cdot \mathrm{s} / \mathrm{deg})$, and $\tau_{\max }$ - the maximum tangential torque exerted while moving along a trajectory $(\mathrm{N} \cdot \mathrm{m})$.

\section{1) Methods}

Three individuals performed the experiment on both devices. The experiment included 128 trials - with 8 movement repetitions along each of the 8 desired trajectories, in two different speed conditions. Speed conditions were defined by different allowable timeframes $(250 \mathrm{~ms}$ to $500 \mathrm{~ms} ; 500 \mathrm{~ms}$ to $750 \mathrm{~ms}$ ) and were cued to the user via visual feedback after trial execution. For each trial, users start in the middle of the workspace and move in the cued direction. Following trial completions, users would return to the middle of the workspace and wait to be cued for the next trial. If a subject did not reach the target within the desired timeframe, they would receive feedback in the form of a green or red target, indicating that

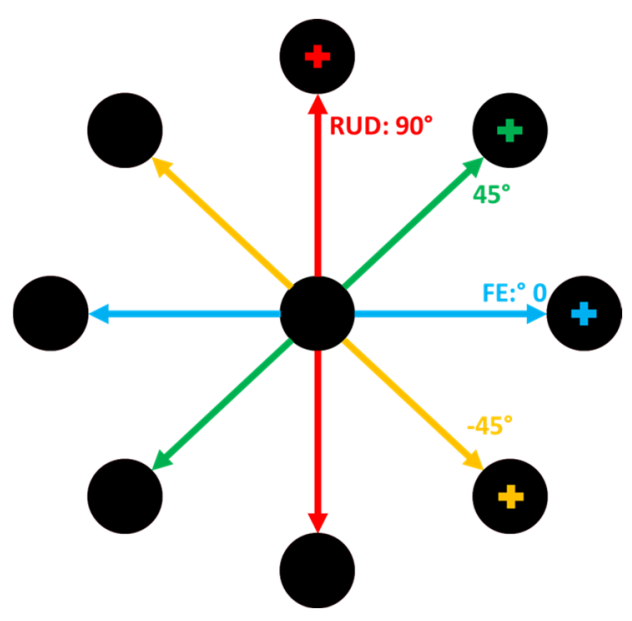

Fig. 6. Trajectories used to investigate dynamic transparency: eight trajectories were examined, classified as positive and negative directions for four paths denoted by their deviation from $0^{\circ}$.

they had gone too fast or too slow, respectively. For postprocessing, trial onset was defined as the instant when the user reached $10 \%$ of that trial's maximum tangential velocity. Trial end was defined as the instant when the user reached the target placed 10 degrees from the start point, with a radial tolerance of 2 degrees. Subjects were told to purposefully overshoot the target such that their maximum tangential velocity would occur near the end of the trial. Both end-effector torques and encoder readings were recorded and converted to measure rotations and torques about the wrist axes, using the methods described in Section I. Data was sampled continuously at $1000 \mathrm{~Hz}$, and velocity profiles were extracted using a Savitzky-Golay filter.

This experiment was done without gravity compensation. As such, to remove the average effect of gravity from the analysis, the time series for both human joint torques (represented by $\tau_{R U D}$ and $\tau_{F E}$ ) were offset by their respective means (across an entire experiment).

To estimate Coulomb friction and viscous friction on each trial, a multiple linear regression was performed using the model in (9):

$$
T=b \dot{\theta}+\tau_{C} \operatorname{sign}(\dot{\theta})+I \ddot{\theta}+A \theta+B
$$

where $\theta$ represents the distance travelled during the task. Trials from two different speed conditions were concatenated to decouple the effects of the positional term from the effects of velocity, and regression was performed on datasets of the resulting pairs of individual trials in the same direction.

To evaluate the effects of the different robots on transparency, a mixed model with two fixed effects (Robot Rob, 2 levels; Direction - Dir, 8 levels) and one random effect (Subject - Sub) was used for each outcome measure $(y)$, i.e., Coulumb friction $\tau_{c}$, viscous friction $b$, and maximum torque $\tau_{\max }$.

$$
\begin{gathered}
y=\beta_{0}+\beta_{1} \cdot R o b+\beta_{2} \cdot \operatorname{Dir}+\beta_{3} \cdot R o b \cdot D i r \\
+\alpha_{0} \cdot S u b
\end{gathered}
$$

\section{2) Results}

Effect of direction is not reported, as the goal of this analysis is to establish the effect of robot on transparency in any direction. As such the post-hoc tests are performed on the average value of the outcome measure $\left(\tau_{C}, b, \tau_{\max }\right)$ across all 
TABLE 3

MiXed MODEL RESUlts: FiXED EFFECTS FOR TRANSPARENCY OUTCOMES

\begin{tabular}{rcccc}
\hline \hline Coulomb Friction & $\mathrm{N}_{\text {parm }}$ & DF $_{\text {Den }}$ & F Ratio & Prob $>$ F \\
Robot & 1 & 366 & 78.861 & $<\mathbf{0 . 0 0 1}$ \\
\hline Robot & 1 & 366 & 0.604 & 0.438 \\
\hline Maximum Torque & $\mathrm{N}_{\text {parm }}$ & $\mathrm{DF}_{\text {Den }}$ & F Ratio & Prob $>\mathrm{F}$ \\
Robot & 1 & 366 & 1.240 & 0.266 \\
\hline \hline
\end{tabular}
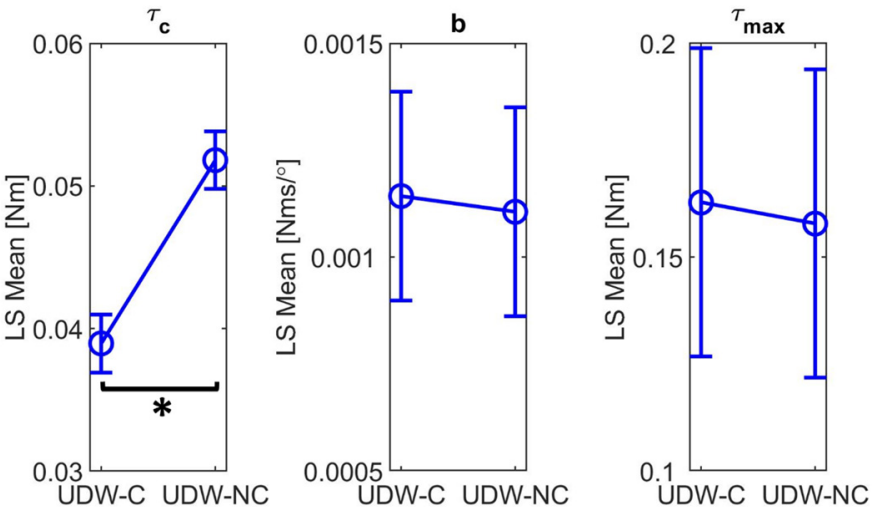

Fig. 7. Mixed model results: effect of robot design. Charts report the estimated least square means and standard error of the means. Asterisk denotes a significant effect $(\mathrm{p}<0.001)$.

directions. The linear mixed model identified a significant effect of factor Rob on Coulomb friction, but not on viscous friction or the maximum torque (Table 3, Fig. 7). Specifically, Coulomb friction is higher in the UDW-NC than in the UDWC (mean and s.e.m.: UDW-C: $0.039 \pm 0.002 \mathrm{Nm}$, UDW-NC: $0.052 \pm 0.002 \mathrm{Nm}$, respectively; $\mathrm{p}<0.001)$.

\section{B. Torque Transmission}

To quantify each system's ability to apply perturbations to the wrist, the systems' end-effectors were rigidly fixed in the neutral configuration $\left(\theta_{R U D}=0 ; \theta_{F E}=0\right.$, Fig. 8). Known torques were commanded to the system and compared to the resulting reaction torques measured via the force-torque sensor located in the end-effector. As the systems are isostatic, constraining the end-effector ensured that all reaction forces were applied through the force sensor in a way that sensor readings corresponded to reactions of the loads being applied to it. Torques were applied in purely FE or RUD and ranged between $\pm 0.5 \mathrm{Nm}$ in increments of $0.1 \mathrm{Nm}$. Commands were held for three seconds and changes in torques were calculated as reactions.

For each command type (pure RUD; pure FE) two outcome measures were used for comparison between the two designs (Fig. 9): reaction torque in the desired direction (i.e., reaction RUD torque for a pure RUD command); and reaction torque in the other DOF (i.e., reaction FE torque for a pure RUD command, due to coupling between the DOFs).

We estimated the slope between the commanded and measured torques for both robot designs via linear regression,
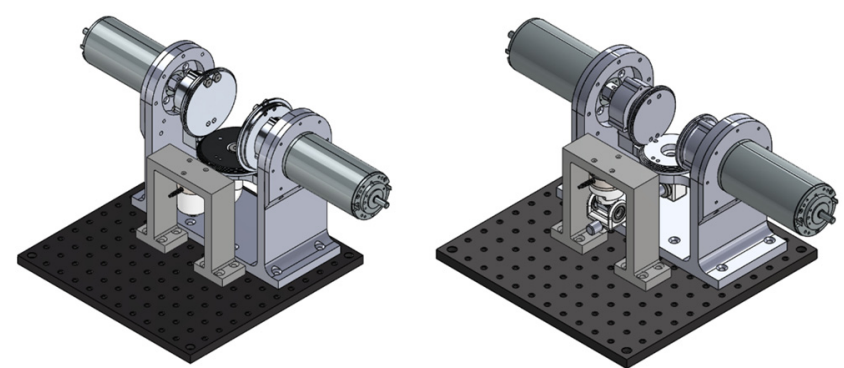

Fig. 8. Apparatus for torque transmission experiment of UDW-C (left) and UDW-NC (right). End-effectors were constrained in such a way that reaction loads were applied through the load sensor.
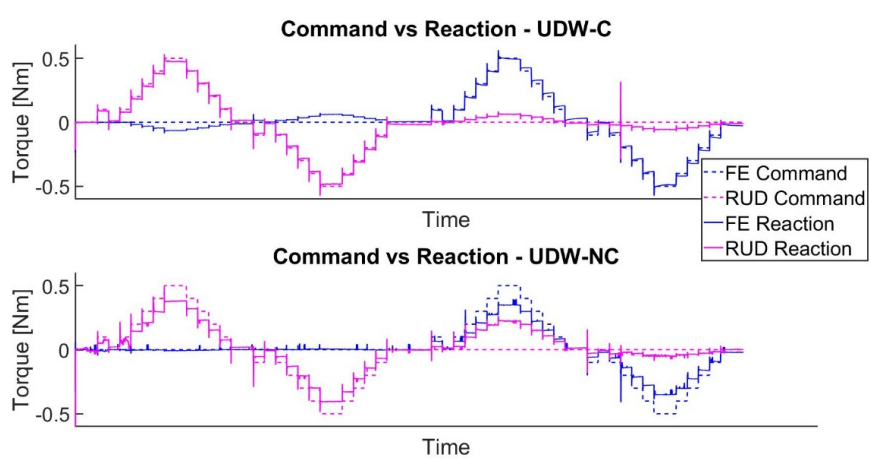

Fig. 9. Torque command vs. reaction for UDW-C (top) and UDW-NC (bottom). Commands were sent in pure RUD and FE, spanning from -0.5 to $+0.5 \mathrm{Nm}$ in increments of $0.1 \mathrm{Nm}$. Commands were held for three seconds.

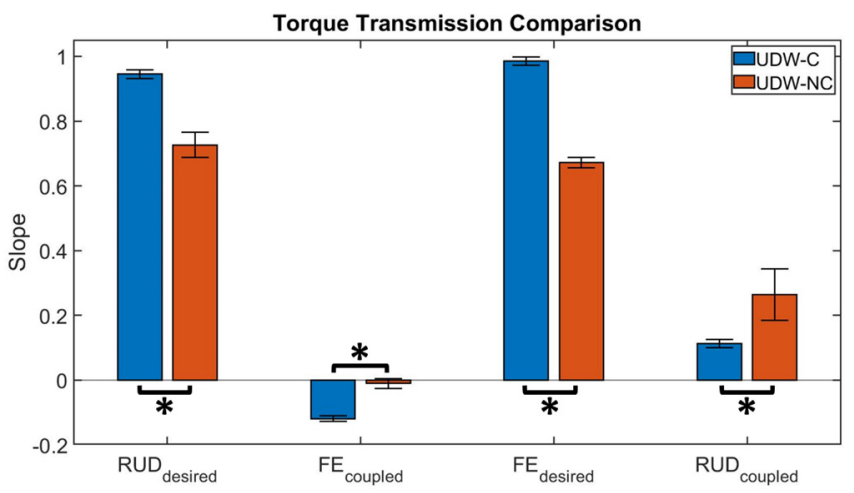

Fig. 10. Slope comparison between systems. Error bars represent $95 \%$ confidence intervals. The asterisks denote significant between-robot differences at $p<0.05$.

and compared these estimates obtained via the different designs to quantify the effects of robot design on torque transmission (Fig. 10). Ideally, the slope should be equal to one for the relationship between commanded and measured torque in the same direction, while it should be equal to zero for the relationship between commanded and measured torque in different directions.

From this analysis, we see that the accuracy of torque transmission is significantly worse in the UDW-NC compared to the UDW-C in the desired direction (FE slope: UDW-NC $0.672 \pm 0.016$, UDW-C $0.986 \pm 0.013$; RUD slope: UDW-NC $0.727 \pm 0.039$, UDW-C $0.945 \pm 0.014)$. Instead, for reactions measured in the non-desired direction, the UDW-NC and UDW-C respond differently in the two directions. Specifically, the UDW-NC shows a very small FE coupling during RUD commands while the coupling is greater for the UDW-C (UDW-NC $-0.010 \pm 0.015$, UDW-C $-0.119 \pm 0.009$ ). 


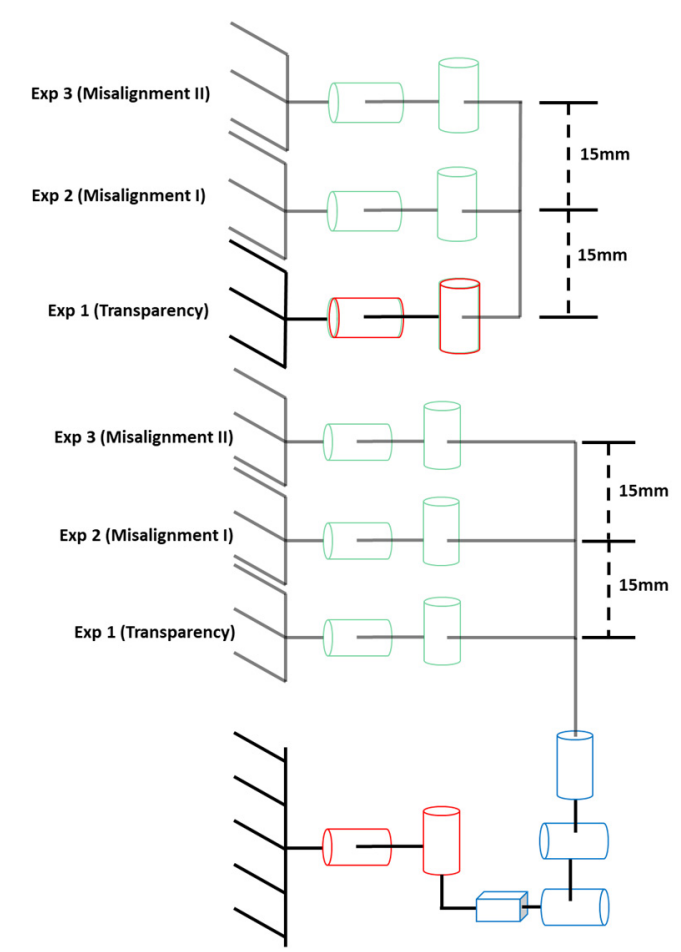

Fig. 11. Misalignment conditions for UDW-C (top) and UDW-NC (bottom) compared to the original transparency experiment: Experimental conditions differ by $15 \mathrm{~mm}$.

Conversely, the UDW-NC displays a greater RUD coupling during FE commands than the UDW-C (UDW-NC $0.264 \pm$ 0.080 , UDW-C $0.113 \pm 0.012$ ).

\section{Effect of Misalignment}

To determine the robustness of each robot design to kinematic misalignment, the transparency experiment was repeated twice more after imposing two different vertical displacements on the user's wrist: $15 \mathrm{~mm}$ and $30 \mathrm{~mm}$ (Fig. 11). These misaligned trials were analyzed in the same manner as the original transparency trials. The dataset comprising these three conditions was subject to a full-factorial mixed model with three fixed effects (Robot - Rob, 3 levels; Direction - Dir, 8 levels; Misalignment - Mis, 3 levels) and one random effect (Subject - Sub), for each outcome measure, as seen in (11).

$$
\begin{gathered}
y=\beta_{0}+\beta_{1} \cdot \text { Rob }+\beta_{2} \cdot \text { Mis }+\beta_{3} \cdot \text { Rob } \cdot \text { Mis } \\
\beta_{4} \cdot \text { Dir }+\beta_{5} \cdot \text { Rob } \cdot \text { Dir }+\beta_{6} \cdot \text { Mis } \cdot \text { Dir } \\
+\beta_{7} \cdot \text { Rob } \cdot \text { Mis } \cdot \text { Dir }+\alpha_{0} \cdot \text { Sub }
\end{gathered}
$$

\section{1) Results}

The linear mixed model identified a significant interaction between factors Rob and Mis on Coulomb friction (Table 4, Fig. 12). This interaction was driven by a greater increase in Coulomb friction associated with the presence of misalignment for the UDW-NC as compared to the UDW-C. Instead, for the higher misalignment condition, the interaction between misalignment and robot design changed direction, and the UDW-C showed a greater increase in Coulomb friction associated with greater misalignments compared to the UDWNC. In fact, the UDW-NC had lower Coloumb friction when going from the low to high misalignment conditions $\left(\Delta \tau_{C}\right.$ between $15 \mathrm{~mm}$ and 0mm: UDW-C: $0.001 \pm 0.001 \mathrm{Nm}$; UDW-
TABLE 4

MiXed Model Results: FiXed EFFECTS FOR MisAlignMENT OUTCOMES

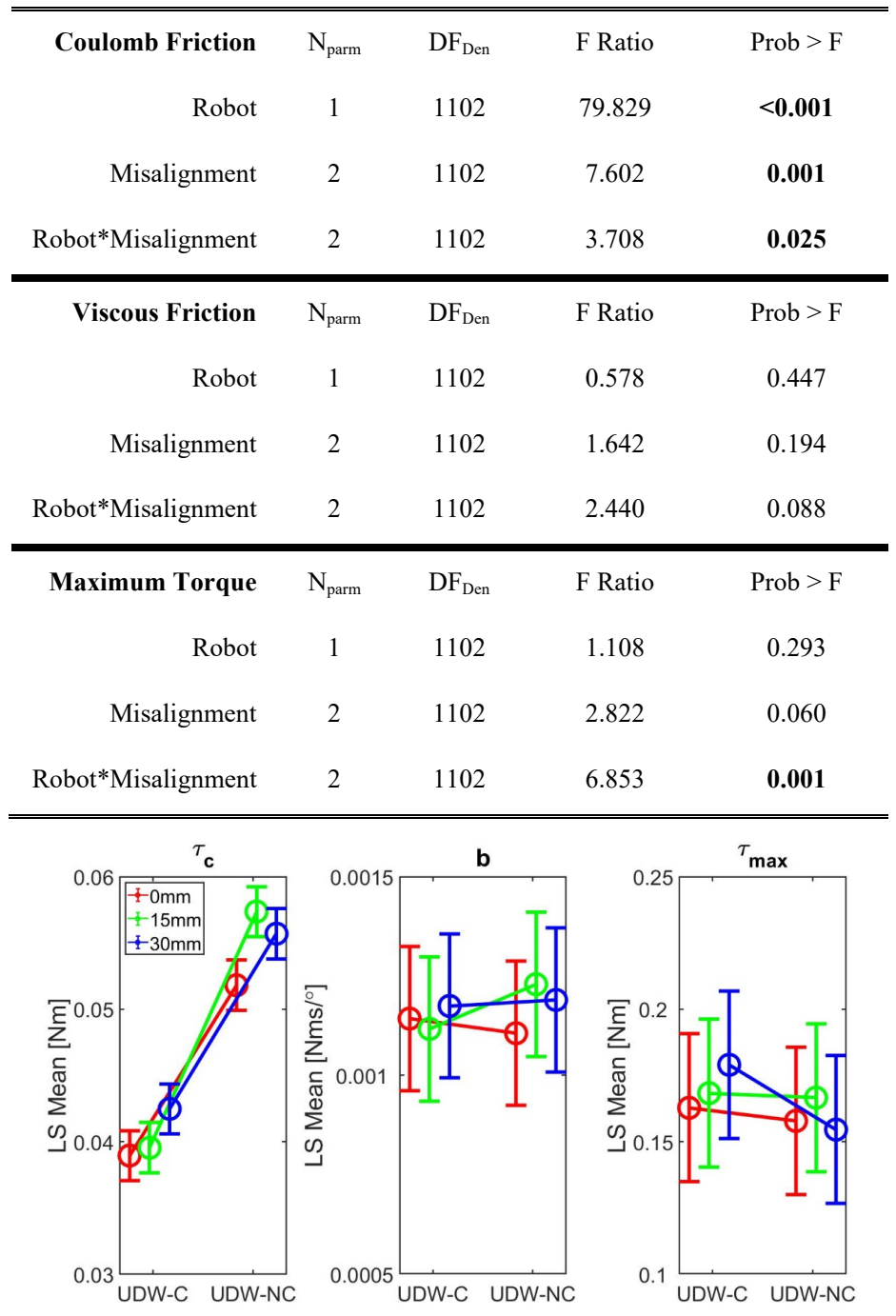

Fig. 12. Mixed model results: two-way interaction $(\mathrm{Rob} \cdot \mathrm{Mis})$. The two-way interaction had a significant effect on both Coulomb friction and maximum torque.
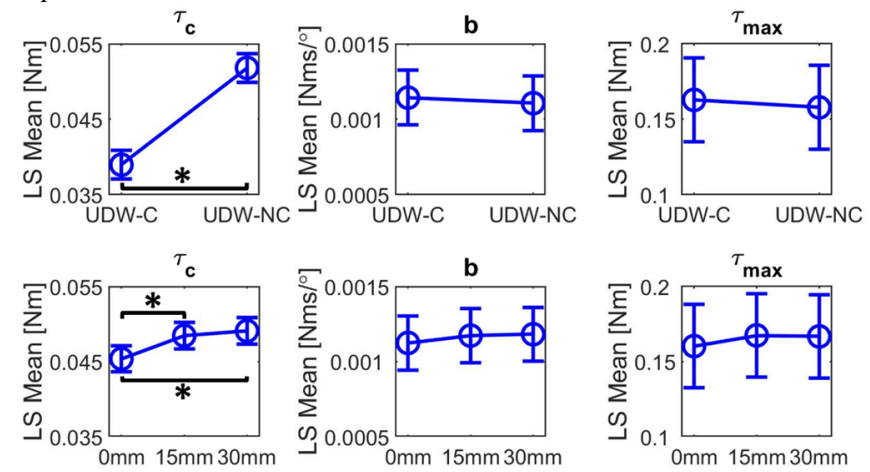

Fig. 13. Mixed model results: effects of Rob (top) and Mis (bottom). Charts report the estimated least square means and standard error of the means. Asterisks indicate significant differences at $p<0.05$.

NC $0.006 \pm 0.001 \mathrm{Nm}$,contrast for the difference between the two $\Delta \tau_{C}$ values $\mathrm{p}=0.015 ; \Delta \tau_{C}$ between $30 \mathrm{~mm}$ and $15 \mathrm{~mm}$ : UDW-C: $0.003 \pm 0.001 \mathrm{Nm}$, UDW-NC: $-0.002 \pm 0.001 \mathrm{Nm}$, contrast $\mathrm{p}<0.001$ ).

For Coulomb friction (Table 4, Fig. 13), the linear mixed 
model further identified a significant effect of robot design (UDW-C: $0.039 \pm 0.002 \mathrm{Nm}$, UDW-NC: $0.052 \pm 0.002 \mathrm{Nm} ; \mathrm{p}$ $<0.001)$, and misalignment (0mm: $0.045 \pm 0.002 \mathrm{Nm} ; \mathbf{1 5 m m}$ : $0.049 \pm 0.002 \mathrm{Nm}$; 30 mm: $0.049 \pm 0.002 \mathrm{Nm}, \mathbf{0 m m}$ vs $15 \mathrm{~mm}$ : $\mathrm{p}=0.001,0 \mathrm{~mm}$ vs 30mm: $\mathrm{p}<0.001)$.

The linear mixed model also identified a significant interaction between robot and misalignment for maximum interaction torque ( $\tau_{\max }$ - Table 4, Fig. 12). This interaction was driven by a smaller increase in interaction torque associated with greater misalignments in the UDW-NC, compared to the increase measured in the UDW-C, for both misalignment conditions $\left(\Delta \tau_{c}\right.$ between $30 \mathrm{~mm}$ and $0 \mathrm{~mm}$ : UDW-C: 0.016 $\pm 0.005 \mathrm{Nm}$; UDW-NC $-0.003 \pm 0.005 \mathrm{Nm}, \mathrm{p}=0.003 ; \Delta \tau_{C}$ between $30 \mathrm{~mm}$ and $15 \mathrm{~mm}$ : UDW-C: $0.011 \pm 0.005 \mathrm{Nm}$, UDW-NC: $-0.012 \pm 0.005 \mathrm{Nm}, \mathrm{p}<0.001$

No significant effect of either factor or of their interaction was found for viscous friction.

\section{DISCUSSION}

In this study, we presented the design and characterization of the UDiffWrist: a 2 DOF wrist exoskeleton which uses a cable differential transmission to achieve low output impedance and ensure backdrivability. Two different versions of the robot were developed to investigate different methods of achieving kinematic compatibility: the UDW-C, featuring a bigger transmission and assuming perfect alignment between user and robot; and the UDW-NC, which incorporates a passive mechanism between human and robotic chains, and thus does not require any alignment. We investigated and quantified the effect of robot design on the transparency of the systems, their ability to transfer torque to the wrist, and their robustness against purposeful misalignments.

Table 5 allows for a comparison between our two systems and other wrist exoskeletons. Via the use of a cable differential transmission, our devices rank favorably in terms of inertia and friction compared to previous wrist robot devices. Specifically, in the FE DOF, our systems have the smallest values of inertia and viscous friction. This is because during $\mathrm{FE}$ the only moving components of the system are the three pulleys (and the endeffector, who's effects are negligible for the UDW-C). In contrast, in RUD, the transmission carriers move, contributing to inertial and friction effects. Thus, the extra inertia and friction associated with the passive mechanism of the UDW-NC contribute to its higher inertia and friction properties.

We observed significant differences in the Coulomb friction of the two systems featuring the two different designs: specifically, Coulomb friction was lower in the UDW-C ( $p<$ 0.001 ). This was unexpected, as the misalignments present in the UDW-C during experimentation were hypothesized to lead to an increase in the end-effector force required to initiate motion. It is possible that for small ranges of motion, such as those required for the tasks performed in this study, these misalignments are not large enough to produce significant consequences on system transparency. It is also possible that any effects caused by the misalignments are offset by the high transparency associated with the cable differential transmission. In fact, as both systems incorporate the same transmission (although smaller in the UDW-NC), it is believed that the passive mechanism implemented in the UDW-NC to release the alignment constraint is what caused the greater friction in that system: the inertia and friction associated with the transmission are low enough such that those associated with the passive mechanism are immediately observable.

The effect of robot design was also found to be significant for torque transmission to the wrist. As seen in Fig. 10, all four desired outcomes are significantly different between the two systems. The UDW-NC was significantly worse at transferring desired torque to the wrist. As discussed in [18] and [41], the implementation of the passive DOFs leads to inefficient load transfer: the joints are incorporated in such a way that under load they displace into a zero-load configuration. As mentioned, this is the same reason why this method is so effective at reducing interaction loads and seems to be an intrinsic drawback on the introduction of passive joints in the kinematic chain of exoskeletons.

We also found an interaction between robot design and level of misalignment for Coulomb friction and maximum interaction torque, which indicates that there was an effect of robot design on each system's robustness to misalignment. Specifically, based on the outcome measure of Coulomb friction, the UDW$\mathrm{NC}$ appeared to be less robust to misalignments in the $15 \mathrm{~mm}$ condition compared to the UDW-C, but such difference was not maintained for the $30 \mathrm{~mm}$ condition. This was not an expected result: as the UDW-NC is an alignment-free robot our expectation was that it would not result in a significant increase in interaction torque, and in a smaller increase compared to the UWD-C, in presence of misalignments. The use of the passive mechanism in the UDW-NC was hypothesized to allow the design to be more robust than the co-located version, based on the studies done in [9] and [17] where the solutions presented allowed usability over the entire workspace and reduced interaction loads. On the other hand, the analysis of results of

TABLE 5

DYNAMIC PARAMETERS OF WRIST ROBOTS

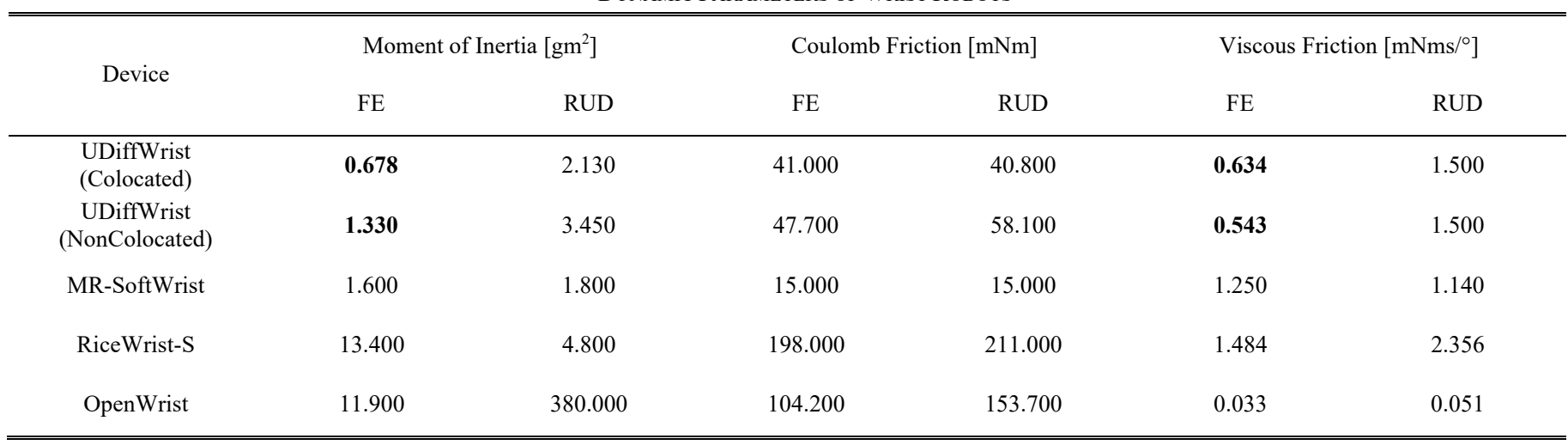


maximum torque indicated that the UDW-NC is indeed more robust to misalignments compared to the UDW-C.

In short, the analysis of results associated with Coulomb friction indicate that the UDW-C is more transparent and robust to misalignments compared to the UWD-NC. In contrast, the analysis of results associated with maximum torque indicate that the UDW-NC is more robust to misalignments compared to the UDW-C. There are two likely sources for such noncoherent results in the two outcome measures. On one hand, Coulomb friction and max torque provide two different indications of the transparency of a robot. Maximum torque is likely a more appropriate outcome measure to study tolerance of a robot in presence of misalignments. In fact, to the authors' knowledge, Coulomb friction has not previously been used as an outcome measure to quantify exoskeleton robustness with respect to misalignment; instead, mean interaction loads and range of motion are used [17], [24], [26], [27]. On the other hand, it is also possible that given the limited range of motion studied in this paper, the misalignment conditions imposed during this experiment were not sufficient to provide a sufficient signal-to-noise ratio to identify effects with enough statistical power. For the analysis of misalignments based on maximum torque, the significant effect only appears for the 30 $\mathrm{mm}$ condition, further indicating that perhaps larger misalignments may be necessary to induce a measurable effect.

From the above results, it is evident that the strength of the UDiffWrist is the cable differential transmission, which allows the system to have minimum inertia, friction, and no backlash. For a 2 DOF joint co-located with the system, the robot can accurately transfer torques to each DOF. The comparison between the two different versions of the robot indicates that in conditions of small misalignments (within $15 \mathrm{~mm}$ ) and small range of motion, the possible benefits of implementing a passive mechanism are smaller than the associated adverse effects on backdrivability. Further, as expected, without proper compensation these extra DOFs lead to inefficient torque transfer.

Based on the UDW-C's ability to transfer torque to specific joints while maintaining minimal coupling torque, as well as the system's high transparency, the design could be implemented as a wrist module for an upper-limb rehabilitation exoskeleton. Depending on the required FE and RUD ROM, the misalignments between user and robot should not warrant the need for the implementation of any passive mechanisms to release the alignment condition.

To allow the user the keep their wrist above the transmission of the UDW-NC, the end-effector of the UDW-NC is larger than the one used for the UDW-C. Even though mean gravitational effects were accounted for by offsetting the entire torque time series, it is possible that there were some residual inertial effects associated with this larger handle that were attributed to Coulomb friction in the performed regressions. Another possible confound in the UDW-NC is the improper fixation of the user's wrist to ground: The UDW-C setup provides a wrist support for the user such that the user is grounded very close to the wrist. However, as the prismatic joint of the UDW-NC can extend behind the robot's transmission, the same wrist support must be further back, allowing the user to perform some movements with a combination of wrist and elbow motion.

To improve upon the UDW-NC design, additional elements can be added into the passive mechanism, (such as the gravity compensating mechanism implemented in [26]) to reduce the effects of the additional mass. However, any design with additional DOFs will inevitably increase the mass of the system. Lightweight materials can also be used in the compensation mechanism to offset inertial effects on reverse drive performance.

In future works, we seek to investigate the interactions between larger misalignments and robot design, as well as if tasks that cover a larger range of motion lead to significant deviations in backdrivability caused by misalignments.

\section{ACKNOWLEDGMENT}

This work was supported by the National Science Foundation under Grant 1943712.

\section{REFERENCES}

[1] A. G. Dunning and J. L. Herder, "A review of assistive devices for arm balancing," IEEE Int. Conf. Rehabil. Robot., 2013, doi: 10.1109/ICORR.2013.6650485.

[2] G. Kwakkel, B. J. Kollen, and H. I. Krebs, "Effects of robot-assisted therapy on upper limb recovery after stroke: A systematic review," Neurorehabil. Neural Repair, vol. 22, no. 2, pp. 111-121, 2008, doi: $10.1177 / 1545968307305457$.

[3] A. Sunderland, D. J. Tinson, E. L. Bradley, D. Fletcher, R. Langton Hewer, and D. T. Wade, "Enhanced physical therapy improves recovery of arm function after stroke. A randomised controlled trial," J. Neurol. Neurosurg. Psychiatry, vol. 55, no. 7, pp. 530-535, 1992, doi: 10.1136/jnnp.55.7.530.

[4] R. Riener, T. Nef, and G. Colombo, "Robot-aided neurorehabilitation of the upper extremities," Med. Biol. Eng. Comput., vol. 43, no. 1, pp. 2-10, 2005, doi: 10.1007/BF02345116.

[5] D. Reinkensmeyer, P. Lum, and J. Winters, "Emerging technologies for improving access to movement therapy following neurologic injury," Emerg. Access. Telecommun. Inf. Healthc. Technol., pp. 1-15, 2002.

[6] H. Barbeau, D. A. McCrea, M. J. O’Donovan, S. Rossignol, W. M. Grill, and M. A. Lemay, "Tapping into spinal circuits to restore motor function," Brain Res. Rev., vol. 30, no. 1, pp. 27-51, 1999, doi: 10.1016/S0165-0173(99)00008-9.

[7] M. et Reinkensmeyer, D J Kahn, L E Averbuch, "Understanding and treating arm movement impairment after chronic brain injury progress with the ARM guide," J. Rehabil. Res. Dev., vol. 37, no. 6, pp. 34-39, 2013.

[8] C. Bütefisch, H. Hummelsheim, P. Denzler, and K. H. Mauritz, "Repetitive training of isolated movements improves the outcome of motor rehabilitation of the centrally paretic hand," J. Neurol. Sci., vol. 130, no. 1, pp. 59-68, May 1995, doi: 10.1016/0022- 
510X(95)00003-K.

[9] A. Schiele and F. C. T. Van Der Helm, "Kinematic Design to Improve Ergonomics in Human Machine Interaction," IEEE Trans. Neural Syst. Rehabil. Eng., vol. 14, no. 4, pp. 456-469, 2006.

[10] R. Colombo et al., "Assessing mechanisms of recovery during robot-aided neurorehabilitation of the upper limb," Neurorehabil. Neural Repair, vol. 22, no. 1, pp. 50-63, 2008, doi: 10.1177/1545968307303401.

[11] N. Norouzi-Gheidari, P. S. Archambault, and J. Fung, "Effects of robot-assisted therapy on stroke rehabilitation in upper limbs: Systematic review and meta-analysis of the literature," J. Rehabil. Res. Dev., vol. 49, no. 4, pp. 479-496, 2012, doi: 10.1682/JRRD.2010.10.0210.

[12] E. B. Mehrholz J, Pohl M, Platz T, Kugler J, "Cochrane Library Cochrane Database of Systematic Reviews Electromechanical and robot-assisted arm training for improving activities of daily living, arm function, and arm muscle strength aer stroke (Review)," 2018, doi: 10.1002/14651858.CD006876.pub5.

[13] T. Nef, M. Mihelj, and R. Riener, "ARMin: A robot for patient-cooperative arm therapy," Med. Biol. Eng. Comput., vol. 45, no. 9, pp. 887-900, 2007, doi: 10.1007/s11517-007-0226-6.

[14] J. C. Perry, J. Rosen, and S. Burns, "Upper-limb powered exoskeleton design," IEEE/ASME Trans. Mechatronics, vol. 12, no. 4, pp. 408-417, 2007, doi: 10.1109/TMECH.2007.901934.

[15] M. B. Naf, K. Junius, M. Rossini, C. RodriguezGuerrero, B. Vanderborght, and D. Lefeber, "Misalignment Compensation for Full HumanExoskeleton Kinematic Compatibility: State of the Art and Evaluation," Appl. Mech. Rev., vol. 70, no. 5, pp. 1-19, 2018, doi: 10.1115/1.4042523.

[16] M. Cempini, S. M. M. De Rossi, T. Lenzi, N. Vitiello, and M. C. Carrozza, "Self-alignment mechanisms for assistive wearable robots: A kinetostatic compatibility method," IEEE Trans. Robot., vol. 29, no. 1, pp. 236250, 2013, doi: 10.1109/TRO.2012.2226381.

[17] N. Jarrassé and G. Morel, "Connecting a human limb to an exoskeleton," IEEE Trans. Robot., vol. 28, no. 3, pp. 697-709, 2012, doi: 10.1109/TRO.2011.2178151.

[18] S. E. Axes and T. Decoupling, "Short Papers of Joint Rotations and Translations," Ieee Trans. Robot., vol. 25, no. 3, pp. 628-633, 2009.

[19] B. Kim and A. D. Deshpande, "An upper-body rehabilitation exoskeleton Harmony with an anatomical shoulder mechanism: Design, modeling, control, and performance evaluation," Int. J. Rob. Res., vol. 36, no. 4, pp. 414-435, 2017, doi: $10.1177 / 0278364917706743$.

[20] T. Nef, M. Guidali, and R. Riener, "ARMin III - arm therapy exoskeleton with an ergonomic shoulder actuation," Appl. Bionics Biomech., vol. 6, no. 2, pp. 127-142, 2009, doi: 10.1080/11762320902840179.
[21] M. Mihelj, T. Nef, and R. Riener, "ARMin II - 7 DoF rehabilitation robot: Mechanics and kinematics," Proc. - IEEE Int. Conf. Robot. Autom., no. April, pp. 41204125, 2007, doi: 10.1109/ROBOT.2007.364112.

[22] M. A. Ergin and V. Patoglu, "ASSISTON-SE : A SelfAligning Shoulder-Elbow Exoskeleton," IEEE Int. Conf. Robot. Autom., pp. 2479-2485, 2012.

[23] B. Celebi, M. Yalcin, and V. Patoglu, "Kinematics and Design of A SSIST O N -SE :," 2013 IEEE/RSJ Int. Conf. Intell. Robot. Syst. Novemb. 3-7, 2013, pp. 15791585, 2013.

[24] B. Dehez and J. Sapin, "ShouldeRO, an alignment-free two-DOF rehabilitation robot for the shoulder complex," IEEE Int. Conf. Rehabil. Robot., pp. 141148, 2011.

[25] D. Galinski, J. Sapin, and B. Dehez, "Optimal design of an alignment-free two-DOF rehabilitation robot for the shoulder complex," IEEE Int. Conf. Rehabil. Robot., 2013, doi: 10.1109/ICORR.2013.6650502.

[26] J. F. Schorsch, A. Q. L. Keemink, A. H. A. Stienen, F. C. T. Van Der Helm, and D. A. Abbink, "A novel selfaligning mechanism to decouple force and torques for a planar exoskeleton joint," Mech. Sci., vol. 5, no. 2, pp. 29-35, 2014, doi: 10.5194/ms-5-29-2014.

[27] A. H. A. Stienen et al., "Dampace: Design of an exoskeleton for force-coordination training in upperextremity rehabilitation," J. Med. Devices, Trans. $A S M E$, vol. 3, no. 3, pp. 1-10, 2009, doi: 10.1115/1.3191727.

[28] D. Zanotto, Y. Akiyama, P. Stegall, and S. K. Agrawal, "Knee Joint Misalignment in Exoskeletons for the Lower Extremities: Effects on User's Gait," IEEE Trans. Robot., vol. 31, no. 4, pp. 978-987, 2015, doi: 10.1109/TRO.2015.2450414.

[29] Krebs et al., "Robot-aided neurorehabilitation: A robot for wrist rehabilitation," IEEE Trans. Neural Syst. Rehabil. Eng., vol. 15, no. 3, pp. 327-335, 2007.

[30] A. Gupta, M. K. O’Malley, V. Patoglu, and C. Burgar, "Design, control and performance of RiceWrist: A force feedback wrist exoskeleton for rehabilitation and training," Int. J. Rob. Res., vol. 27, no. 2, pp. 233-251, 2008, doi: 10.1177/0278364907084261.

[31] A. Erwin, M. K. O’Malley, D. Ress, and F. Sergi, "Kinesthetic Feedback during 2DOF Wrist Movements via a Novel MR-Compatible Robot," IEEE Trans. Neural Syst. Rehabil. Eng., vol. 25, no. 9, pp. 14891499, 2017, doi: 10.1109/TNSRE.2016.2634585.

[32] L. Masia, M. Casadio, P. Giannoni, G. Sandini, and P. Morasso, "Performance adaptive training control strategy for recovering wrist movements in stroke patients: A preliminary, feasibility study," J. Neuroeng. Rehabil., vol. 6, no. 1, pp. 1-11, 2009, doi: 10.1186/1743-0003-6-44.

[33] A. U. Pehlivan, F. Sergi, A. Erwin, N. Yozbatiran, G. E. Francisco, and M. K. O'Malley, "Design and validation of the RiceWrist-S exoskeleton for robotic 
rehabilitation after incomplete spinal cord injury," Robotica, vol. 32, no. 8, pp. 1415-1431, 2014, doi: 10.1017/S0263574714001490.

[34] E. Pezent, C. G. Rose, A. D. Deshpande, and M. K. O'Malley, "Design and characterization of the OpenWrist: A robotic wrist exoskeleton for coordinated hand-wrist rehabilitation," IEEE Int. Conf. Rehabil. Robot., pp. 720-725, 2017, doi: 10.1109/ICORR.2017.8009333.

[35] D. Buongiorno, E. Sotgiu, D. Leonardis, S. Marcheschi, M. Solazzi, and A. Frisoli, "WRES: A Novel 3 DoF WRist ExoSkeleton with Tendon-Driven Differential Transmission for Neuro-Rehabilitation and Teleoperation," IEEE Robot. Autom. Lett., vol. 3, no. 3, pp. 2152-2159, 2018, doi: 10.1109/LRA.2018.2810943.

[36] W. T. Townsend, "United States patent," Geothermics, vol. 14, no. 4, pp. 595-599, 1985, doi: 10.1016/03756505(85)90011-2.

[37] A. Parmiggiani et al., "The design of the iCub humanoid robot," Int. J. Humanoid Robot., vol. 9, no. 4, 2012, doi: 10.1142/S0219843612500272.

[38] F. Focacci, M. Piccigallo, O. Tonet, G. Megali, A. Pietrabissa, and P. Dario, "Lightweight hand-held robot for laparoscopic surgery," Proc. - IEEE Int. Conf. Robot. Autom., no. April, pp. 599-604, 2007, doi: 10.1109/ROBOT.2007.363052.

[39] T. Chen, R. Casas, and P. S. Lum, "An Elbow Exoskeleton for Upper Limb Rehabilitation with Series Elastic Actuator and Cable-Driven Differential," IEEE Trans. Robot., vol. 35, no. 6, pp. 1464-1474, 2019, doi: 10.1109/TRO.2019.2930915.

[40] J. P. Whitney and J. K. Hodgins, "A passively safe and gravity-counterbalanced anthropomorphic robot arm," Proc. - IEEE Int. Conf. Robot. Autom., vol. 6168, no. 3, pp. 6168-6173, 2014, doi: 10.1109/ICRA.2014.6907768.

[41] A. Zonnino and F. Sergi, "Optimal design of cable differential actuation for 2-DOF wrist robots : effect of joint misalignments on interaction force," Eng. Med. Biol. Conf. 2016, Late Break. Res. Submission, vol. 14, no. 2006, p. 5207114, 2016.

[42] D. Campolo, D. Formica, E. Guglielmelli, and F. Keller, "Kinematic analysis of the human wrist during pointing tasks," Exp. Brain Res., vol. 201, no. 3, pp. 561-573, 2010, doi: 10.1007/s00221-009-2073-1.

[43] L. Leonard, D. Sirkett, G. Mullineux, G. E. B. Giddins, and A. W. Miles, "Development of an in-vivo method of wrist joint motion analysis," Clin. Biomech., vol. 20, no. 2, pp. 166-171, 2005, doi: 10.1016/j.clinbiomech.2004.09.005. 